\title{
Approaching the "Lab in the Gap": First Results from a Versatile In-situ (S)TEM
}

\author{
Felix Börrnert ${ }^{1,2,4}$, Heiko Müller ${ }^{3}$, Martin Linck $^{3}$, Alexander Horst ${ }^{2}$, Angus I. Kirkland ${ }^{4}$, \\ Bernd Büchner ${ }^{2}$, and Hannes Lichte ${ }^{1}$ \\ 1. Speziallabor Triebenberg, Technische Universität Dresden, 01062 Dresden, Germany. \\ 2. IFW Dresden, PF 270116, 01171 Dresden, Germany. \\ 3. CEOS GmbH, Englerstraße 28, 69126 Heidelberg, Germany. \\ 4. Department of Materials, University of Oxford, Parks Road, Oxford OX1 3PH, United Kingdom.
}

In the past, the general development of microscopes was governed by the desire for ever higher resolution in the micrographs. This led to programs like the TEAM project resulting in as fantastic machines as the TEAM I microscope offering a resolving power of about half an Ångström in both TEM and STEM mode [1]. In this course, virtually all means were taken to increase the resolving power even if it put severe limitations to other aspects of microscope aided research. As the most prominent examples in (S)TEM serve the millimeter-sized pole piece gap and the few-Tesla-scale magnetic lens field the sample resides in. A recent survey of the DOE in the future needs of microscopy research resulted in a significant change of direction; besides multi-dimensional and ultrafast microscopy the "lab in the gap" approach has been identified as the most promising development in future electron microscopy [2]. To this end, the key development is increasing the space around the sample for multimodal in-situ experiments.

In this contribution, we present a fully operational prototype transmission electron microscope offering a sample chamber with a $70 \mathrm{~mm}$ "pole piece gap" that is accessible through five arbitrarily usable ports ISO-K DN63 from several sides, see Figure 1. Additionally, the sample space is completely magnetic field free. Conceivable configurations are, for example, a tube with a diameter of slightly more than $60 \mathrm{~mm}$ could be driven horizontally through the entire microscope column; with a differential pumping system enabling operation in gaseous environments. Another example is the possible combination of a liquid helium continuous flow cryostat providing long-time stable low temperatures and mobile electrical probers for in-situ transport measurements on structures found and controlled ad hoc in the microscope.

To achieve a decent resolving power with this large sample space, the microscope is operated in a $C_{s}$ corrected Lorentz mode for both conventional and scanning imaging mode. The resolving power of the instrument is about $1 \mathrm{~nm}$ in conventional imaging mode and better than $5 \mathrm{~nm}$ in scanning mode at $200 \mathrm{kV}$ electron acceleration voltage [3]. An upgrade of the electron optics that enhances the microscope's resolving power in conventional imaging mode to $c a .0 .5 \mathrm{~nm}$ is planned for the near future. It is noteworthy that all of the alterations to the original state of the microscope, a double $C_{s}$ corrected JEOL JEM-2010F, are fully reversible. To aid navigation on sample positions that are not electron transparent, e. $g$. for placing mobile probers on pre-processed contact pads, we installed a secondary electron detector. For the mapping of electric and magnetic fields at the nanoscale via off-axis electron holography, an electrostatic biprism is available.

For the design of new experiments to be performed inside the microscope, we built a testing rack with a copy of the microscope's new sample chamber. Also, the rack has the same outer shape as the microscope with its peripherals, thus, all necessary attachments can be checked for collisions and space 
requirements.

The main area of research the microscope is planned for is solid state physics. Examples are in-situ characterization and manipulation of unconventional superconductors, magnetic nanostructures, and topological phenomena. However, this does not exclude any other experiment conceivable for this microscope [4].

References:

[1] P Ercius et al, Microsc. Microanal. 18 (2012), p. 676.

[2] Report of the Basic Energy Sciences Workshop on the Future of Electron Scattering \& Diffraction (2014), URL http://science.energy.gov/ /media/bes/pdf/reports/files/Future_of_Electron_Scattering.pdf. [3] F Börrnert et al, Ultramicrosc. (2015), DOI 10.1016/j.ultramic.2014.11.011.

[4] The authors acknowledge financial support from the European Union under the Seventh Framework Programme under a contract for an Integrated Infrastructure Initiative (Reference 312483-ESTEEM2).
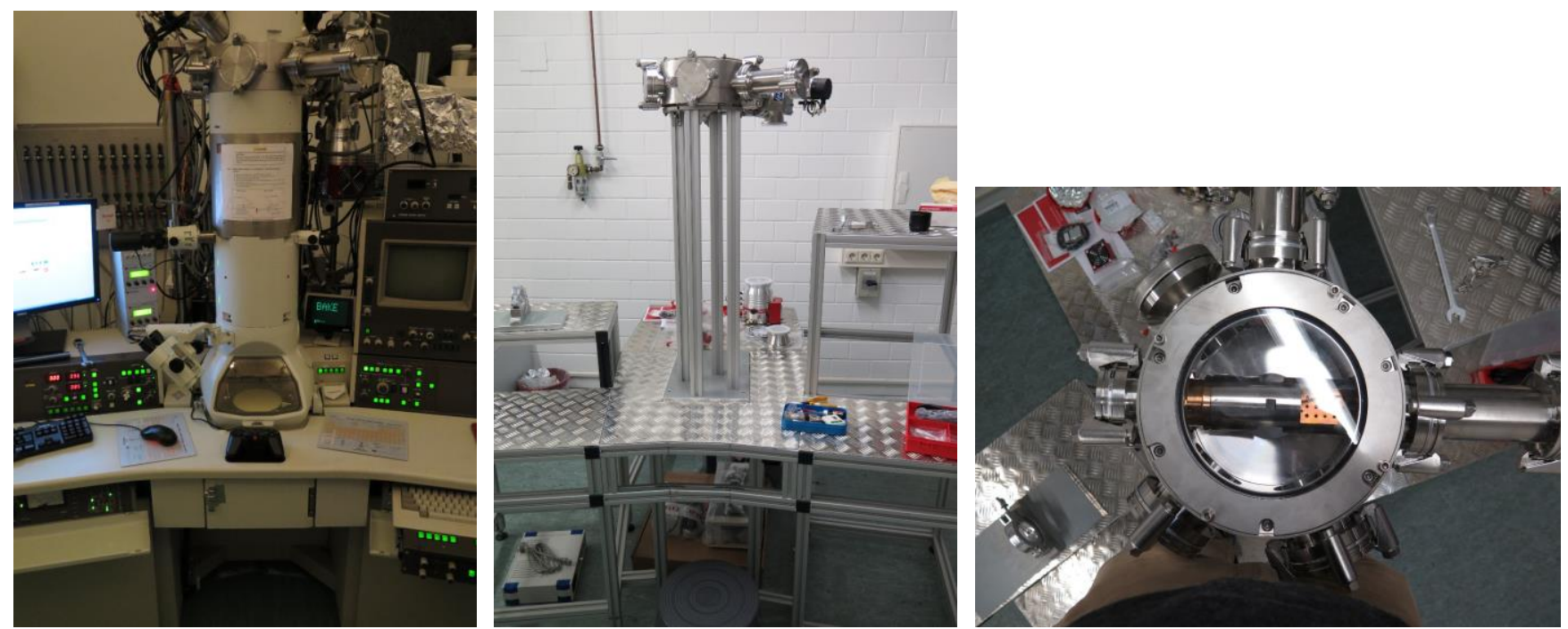

Figure 1. Microscope with new sample chamber, test rack with sample chamber, and sample chamber on test rack viewed along electron beam direction 